\title{
Los estudiantes con discapacidad en la Universidad de Almería: Ideas y Actitudes sobre su integración Educativa y Social
}

\author{
Antonio Sánchez y José J. Carrión \\ Universidad de Almería (España)
}

\begin{abstract}
Hemos planteado como objetivo general hacer una investigación sobre la integración educativa y social de los estudiantes con necesidades especiales asociadas a una discapacidad, desde las perspectivas del personal docente e investigador, del personal de administración y servicios, de los estudiantes en general y de los estudiantes con discapacidad, en la Universidad de Almería. En este artículo, por razones de espacio, sólo recogemos parte de la información referida a los estudiantes con discapacidad que cursan estudios en la Universidad de Almería, quedando nuestro objetivo general formulado en los siguientes términos, "estudiar la percepción y el grado de satisfacción que tienen los estudiantes con discapacidad sobre su integración educativa y social en la Universidad de Almería". Metodológicamente nos situamos en el paradigma naturalista debido a la necesidad de hacer un análisis en y desde la realidad que genera el objeto de estudio, sin manipulación ni alteración de las distintas circunstancias, materiales y personales. Se trata de un diseño de tipo descriptivo y de modalidad selectiva, que utiliza el método de encuesta y como instrumento el cuestionario. Aunque en este artículo finalizamos con algunas de las conclusiones más significativas, hay que tener presente que la profundidad de la investigación permite valorar otras no contempladas y que el lector podrá detectar.

Palabras clave: Educación inclusiva, educación especial, atención educativa a la diversidad, Universidad, discapacidad.
\end{abstract}

Students with disabilities in the University of Almería: ideas and attitudes about your educational and social integration. Our study is an inquiry into the educational and social integration of university students with special needs derived from their disabilities. We investigate how this integration is perceived from the perspectives of teaching / research staff, administration / services personnel, as well as students, including those with disabilities. In this article, for reasons of space, we only collect some information relating to disabled students studying in the University of Almeria, our overall objective being formulated as follows, "to study the perception and degree of satisfaction for students with disabilities on educational and social integration at the University of Almería". Methodologically we stem from the naturalistic paradigm because of the need to accomplish an examination deeply rooted in the reality that generates the object of study, without manipulation or alteration of different circumstances, either material or personal, as well as the perception that have different areas. Thus, we have developed a descriptive design of a selective type which uses the survey method and the questionnaire as its main instrument. Though we conclude with some significant findings, it is noteworthy that many other arguments could be considered in the light of the width and depth of the study, some of which can be identified by the reader.

Key words: Inclusive education, special education, educational treatment of diversity, University, disability.

Correspondencia: Antonio Sánchez Palomino. Departamento de Didáctica y Organización Escolar. Universidad de Almería. Cañada de San Urbano, s/n. C.P. 04120. Almería (España). E-mail: asanchez@ual.es 
En la normativa vigente sobre educación de las personas con discapacidad, destacan los derechos a la no discriminación y a la igualdad de oportunidades. Derechos asumidos por las Naciones Unidas a través del Programa de Acción Mundial para las Personas con Discapacidad (1983), y de las Normas Uniformes sobre la Igualdad de Oportunidades de las Personas con Discapacidad (1993). El Consejo de Europa mediante la Carta Social Europea (1996) y el Tratado de la Unión Europea (Amsterdam, 1997). En el Estado Español también hay que hacer mención a la Ley de Integración Social de los Minusválidos (1982), la Ley Orgánica de Universidades (6/2001, de 21 de diciembre), a la Ley de Igualdad de Oportunidades y No Discriminación de las Personas con Discapacidad (2003), así como a las recientes Ley Orgánica de Educación, 2/2006, de 3 de mayo y a la Ley de Educación de Andalucía, 17/2007 de 10 de diciembre.

El esfuerzo que se está produciendo en el contexto europeo hacia políticas más sociales tiene repercusiones en nuestro país a partir de los años ochenta, comenzando con el cambio llevado a cabo en todo el sistema educativo y concretamente con la promulgación y el posterior desarrollo normativo del Plan Nacional para la Educación Especial, 1978; Ley de Integración Social de los Minusválidos, 1982; Real Decreto de Ordenación de la Educación Especial, 1985; Ley Orgánica de Ordenación General del Sistema Educativo, 1990; Ley Orgánica de la Participación, la Evaluación y el Gobierno de los Centros Docentes, 1990; Real Decreto de Ordenación de la Educación de los Alumnos con Necesidades Educativas Especiales, 1995. Del mismo modo hay que hacer notar, que se han producido significativos avances en la Comunidad Autónoma de Andalucía, que desde el aspecto normativo se concretan en: Ley de Atención a las Personas con Discapacidad, Ley de Solidaridad en la Educación, Decreto sobre Atención al Alumnado con Necesidades Educativas Especiales, Orden de Evaluación Psicopedagógica, Orden sobre Proyectos Curriculares en Centros Específicos de Educación Especial y Orden sobre Programas Transición Vida Adulta y Laboral.

Los cambios desarrollados en el ámbito de la enseñanza no universitaria pronto la transciende llegando a la universidad, ya que al alumnado con discapacidad se le brinda la posibilidad de desarrollarse en contextos más favorecedores del aprendizaje, por lo que la institución universitaria tiene ante sí, un nuevo reto que abordar, para el que debe adoptar los mismos principios que en su día asumieron otros niveles del sistema educativo, esto es, el principio de normalización (referido al entorno socioeducativo), el principio de integración (aceptando la diversidad y la igualdad de oportunidades) y el principio de individualización (atendiendo a las necesidades específicas, especificidad).

En nuestro país se han iniciado distintas experiencias que suponen un reto para la institución universitaria, aunque no son suficientes, debido a que estamos ante una demanda social dinamizada por la integración en los diferentes niveles del sistema educativo y que exige la normalización de la integración en la universidad. 
El objetivo que nos hemos planteado consiste en realizar un estudio acerca de las ideas y actitudes que poseen los estudiantes con discapacidad sobre su integración en la Universidad y, concretamente, analizar la percepción y el grado de satisfacción que tienen sobre su integración educativa y social en la Universidad de Almería.

\section{METODO}

\section{Participantes}

Sobre una población constituida por 138 estudiantes, se identificaron como personas con discapacidad, y por consiguiente solicitaron ayuda de la Unidad de Apoyo al Estudiante con Discapacidad de la Universidad de Almería, un total de 36 personas. Obligados por la ley de privacidad y por los principios éticos que rigen la investigación educativa, hemos de respetar a ese colectivo que desea permanecer en el anonimato y hacer referencia como población invitada a 36 estudiantes, de los que un total de 33 constituyeron la muestra participada, en términos estadísticos prácticamente la totalidad del conglomerado.

En cuanto a la variable edad, la media es de 26.12 años, si tenemos presente que la desviación típica es de 6.5, se sitúa el intervalo de edad entre los 20 y los 49 años. Con respecto al género hay que destacar que el $56 \%$ son mujeres, sin olvidar que 8 de los encuestados son casos perdidos ya que no han contestado al respecto.

Tabla 1. Centros de Estudios

\begin{tabular}{lcr}
\hline & Frecuencia & $\%$ \\
\hline Ciencias de la Salud & 6 & 18.2 \\
Ciencias Económicas y Empresariales & 9 & 27.3 \\
Ciencias Experimentales & 1 & 3.0 \\
Derecho & 2 & 6.1 \\
Humanidades y Ciencias de la Educación & 12 & 36.4 \\
Politécnica Superior & 3 & 9.1 \\
Total & 33 & 100 \\
\hline
\end{tabular}

Tabla 2. Tipo de discapacidad

\begin{tabular}{lll}
\hline & Frecuencia & $\%$ \\
\hline Física & 16 & 48.5 \\
Psíquica & 4 & 12.1 \\
Auditiva & 2 & 6.1 \\
Visual & 3 & 9.1 \\
Otras & 5 & 15.2 \\
Física y otras & 3 & 9.0 \\
Total & 33 & 100 \\
\hline
\end{tabular}

Otra de las variables a considerar es la carrera o titulación que cursan los estudiantes con discapacidad, la mayoría de los cuales se encuentran en la Facultad de Humanidades y Ciencias de la Educación. 
En relación con el tipo de discapacidad que presentan, en el conjunto de la muestra prevalece la discapacidad física, tal como se muestra en la siguiente tabla.

\section{Instrumento}

Los ítems que integran el cuestionario se agrupan en dos factores teóricos, ideas y actitudes, que podemos identificar en el siguiente cuadro (ver Anexo I).

Tabla 3. Estructura del cuestionario según las ideas y actitudes

\begin{tabular}{cc}
\hline IDEAS & ACTITUDES \\
\hline $1,2,3,4,5,7,8,9,10,11,12,13,14,15,16,17,18,19,20,21,22$, & \multirow{2}{*}{6 y 36} \\
$23,24,25,26,27,28,29,30,31,32,33,34,35,37,38,39$ y 40 & \\
\hline
\end{tabular}

Son factores teóricos las ideas y las actitudes que tienen los estudiantes con discapacidad de la Universidad de Almería, en relación con la percepción y grado de satisfacción sobre su integración educativa y social.

Las categorías en las respuestas a los ítems del cuestionario se establecieron a partir de la pregunta inicial "con respecto a los estudiantes de la Universidad de Almería que presentan algún tipo de discapacidad, pienso que” (tache con una X la puntuación más adecuada en función del grado de acuerdo-desacuerdo), de manera que en el análisis y valoración de los resultados más significativos de los factores teóricos ideas y actitudes, hemos establecido dos categorías en relación con las respuestas a los ítems del cuestionario: En desacuerdo (rechazo): que se corresponde con las categorías 1 (totalmente en desacuerdo) y 2 (en desacuerdo); y De acuerdo (aceptación): categoría referida a las respuestas 3 (de acuerdo) y 4 (totalmente de acuerdo).

\section{Procedimiento}

Para la obtención de datos hemos utilizado como técnica de investigación la encuesta, que mediante cuestionarios se ha aplicado a los cuatro ámbitos o conglomerados objeto de estudios, esto es, al personal docente e investigador (PDI), al personal de administración y servicios (PAS), a los estudiantes en general y a los estudiantes con discapacidad, conglomerados que integran la población de la comunidad académico educativa de la Universidad de Almería.

En relación a los estudiantes con discapacidad (ED), la población invitada abarca la totalidad de la población objeto de estudio, llegando de esta forma a cubrir el cien por cien de los sujetos que la integran. De manera que no hemos realizado proceso alguno de muestreo ni de representatividad de la muestra, operando sobre una muestra participada o aceptante productora de datos (Fox, 1981), a la que aplicándole el test de validez muestral obtenemos un nivel de confianza del $95 \%$ y un margen de error inferior al $10 \%$ en todos los ámbitos. 


\section{RESULTADOS}

Presentamos a continuación el análisis y valoración de los resultados más significativos en relación a los factores teóricos ideas y actitudes a partir de los ítems del cuestionario.

Factores teóricos: ideas y actitudes

En la siguiente tabla mostramos los y porcentajes de respuesta que existen en el conjunto de los ítems agrupados en torno a los factores teóricos ideas y actitudes.

Tabla 4. Frecuencias y porcentajes de respuestas en los factores teóricos

\begin{tabular}{cccccc}
\hline \multicolumn{2}{c}{ FACTORES TEÓRICOS } & 1 & 2 & 3 & 4 \\
\hline IDEAS & Porcentajes & 20.0 & 10.1 & 21.5 & 48.4 \\
\hline ACTITUDES & Porcentajes & 13.6 & 9.1 & 33.3 & 43.9 \\
\hline
\end{tabular}

Como podemos observar, en relación con el factor teórico ideas, existe sólo un $69.9 \%$ de respuestas dadas por los estudiantes con discapacidad, que hacen referencia a la aceptación y sensibilidad sobre su integración en la Universidad, ya que el resto $(30.1 \%)$ se sitúan en una visión negativa y de rechazo. De forma similar, podemos encontrar también en el factor actitudes, aunque con una tendencia ligeramente superior en cuanto a su percepción y grado de satisfacción sobre la integración de los estudiantes con discapacidad en la Universidad. En efecto, existe un $77.2 \%$ de respuestas que indican una predisposición positiva hacia la integración, mientras que sólo el $22.7 \%$ de las respuestas se encuentran en el extremo opuesto. Atendiendo a estos datos, podemos deducir a grandes rasgos que existe una tendencia positiva y de aceptación en cuanto a la percepción y el grado de satisfacción por parte de los estudiantes con discapacidad sobre su integración en la Universidad. No obstante, dada la importancia que tiene la especificidad y la singularidad del contenido enunciado en cada ítem, de una parte, y, de otra, teniendo en cuenta la diversidad de respuestas existentes, es necesario profundizar en el análisis de cada ítem. A continuación, exponemos los datos más significativos utilizando para ello, los estadísticos descriptivos tales como los porcentajes de respuesta, la media y la desviación típica.

En relación con el factor teórico ideas, podemos observar que en primer lugar destaca la idea de que es necesario que la universidad firme convenios de colaboración con Asociaciones e Instituciones Públicas y/o Privadas para favorecer una mejora de la atención hacia los estudiantes con discapacidad (ítem 33), con una media de 3.79 y una desviación típica de 0.41 , que pone de manifiesto la poca dispersión o variabilidad en las respuestas, siendo el $100 \%$ de los encuestados los que aceptan esta idea.

En la misma línea se manifiestan en el ítem 32, al afirmar el 94\% de los encuestados, con una media de 3.78 y una desviación típica de 0.49 , que debe existir una 
unidad central que coordine y asesore a estudiantes con discapacidad, al profesorado y al personal de administración y servicios.

Tabla 5. Porcentajes de respuesta, medias y desviaciones típicas del factor ideas

\begin{tabular}{|c|c|c|c|c|c|c|}
\hline Ítems: Factor Teórico Ideas & 1 & 2 & 3 & 4 & $M$ & D.T \\
\hline 1 & 66.7 & 18.2 & 15.2 & 0 & 1.48 & 0.75 \\
\hline 2 & 39.4 & 18.2 & 0 & 42.4 & 2.45 & 1.39 \\
\hline 3 & 9.1 & 0 & 15.2 & 75.8 & 3.58 & 0.90 \\
\hline 4 & 51.5 & 18.2 & 21.2 & 9.1 & 1.88 & 1.05 \\
\hline 5 & 3.0 & 0 & 12.1 & 84.8 & 3.79 & 0.60 \\
\hline 7 & 21.2 & 9.1 & 6.1 & 63.6 & 3.12 & 1.27 \\
\hline 8 & 39.4 & 9.1 & 36.4 & 15.2 & 2.27 & 1.15 \\
\hline 9 & 27.3 & 30.3 & 12.1 & 30.3 & 2.45 & 1.20 \\
\hline 10 & 63.6 & 36.4 & 0 & 0 & 1.36 & 0.49 \\
\hline 11 & 30.3 & 15.2 & 6.1 & 48.5 & 2.73 & 1.35 \\
\hline 12 & 63.6 & 36.4 & 0 & 0 & 1.36 & 0.49 \\
\hline 13 & 66.7 & 18.2 & 9.1 & 6.1 & 1.55 & 0.90 \\
\hline 14 & 6.1 & 0 & 24.2 & 69.7 & 3.58 & 0.79 \\
\hline 15 & 21.2 & 9.1 & 12.1 & 57.6 & 3.06 & 1.25 \\
\hline 16 & 60.6 & 3 & 15.2 & 21.2 & 1.97 & 1.29 \\
\hline 17 & 69.7 & 12.1 & 12.1 & 6.1 & 1.55 & 0.94 \\
\hline 18 & 63.6 & 24.2 & 6.1 & 6.1 & 1.55 & 0.87 \\
\hline 19 & 45.5 & 24.2 & 3 & 27.3 & 2.12 & 1.27 \\
\hline 20 & 36.4 & 24.2 & 15.2 & 24.2 & 2.27 & 1.21 \\
\hline 21 & 69.7 & 21.2 & 9.1 & 0 & 1.39 & 0.66 \\
\hline 22 & 27.3 & 15.2 & 15.2 & 42.4 & 2.73 & 1.28 \\
\hline 23 & 30.3 & 18.2 & 6.1 & 45.5 & 2.67 & 1.34 \\
\hline 24 & 42.4 & 18.2 & 18.2 & 21.2 & 2.18 & 1.21 \\
\hline 25 & 30.3 & 21.2 & 39.4 & 9.1 & 2.27 & 1.01 \\
\hline 26 & 0 & 21.2 & 36.4 & 39.4 & 3.19 & 0.78 \\
\hline 27 & 0 & 3.0 & 51.5 & 45.5 & 3.42 & 0.56 \\
\hline 28 & 45.5 & 12.1 & 18.2 & 24.2 & 2.21 & 1.27 \\
\hline 29 & 12.1 & 24.2 & 51.5 & 12.1 & 2.64 & 0.86 \\
\hline 30 & 6.1 & 18.2 & 54.5 & 18.2 & 2.88 & 0.79 \\
\hline 31 & 12.1 & 6.1 & 18.2 & 63.6 & 3.33 & 1.05 \\
\hline 32 & 0 & 3.0 & 15.2 & 78.8 & 3.78 & 0.49 \\
\hline 33 & 0 & 0 & 21.2 & 78.8 & 3.79 & 0.41 \\
\hline 34 & 9.1 & 3.0 & 12.1 & 75.8 & 3.55 & 0.94 \\
\hline 35 & 3.0 & 3.0 & 18.2 & 69.7 & 3.65 & 0.71 \\
\hline 37 & 36.4 & 27.3 & 12.1 & 24.2 & 2.24 & 1.20 \\
\hline 38 & 15.2 & 0 & 18.2 & 60.6 & 3.32 & 1.11 \\
\hline 39 & 0 & 6.1 & 18.2 & 75.8 & 3.70 & 0.58 \\
\hline 40 & 30.3 & 6.1 & 6.1 & 54.5 & 2.88 & 1.38 \\
\hline
\end{tabular}

Asimismo, el 94\% de los estudiantes con discapacidad aceptan la idea de que se deben incrementar los esfuerzos económicos para mejorar la atención educativa que reciben (ítem 39). No obstante, teniendo en cuenta el valor de la desviación típica (0.58), hay que señalar que el $6.1 \%$ afirman no estar de acuerdo con esta idea.

También destacan las respuestas al ítem 5, con una media de 3.79 y una desviación de 0.60 , para quienes el $96.9 \%$ piensan que es necesario asegurar la igualdad de oportunidades en el acceso a la universidad. Sin embargo, llama la atención el hecho de que un $3 \%$ de los encuestados se manifiesten en sentido contrario, rechazando totalmente el principio de igualdad de oportunidades. 
Las respuestas al ítem 35 muestran, con una media de 3.65 y una desviación de 0.71 , lo que indica una significativa dispersión en las respuestas, que el $87.9 \%$ de los encuestados piensan que la titulación alcanzada por los estudiantes con discapacidad debe ser garante del conjunto de competencias necesarias para su ejercicio profesional. Hecho que contrasta con que el otro $6 \%$ no estén de acuerdo con esta idea.

Asimismo, en relación con el ítem 14, hay que señalar que el 93.9\% de los estudiantes con discapacidad afirman la importancia de fomentar actitudes favorables hacia ellos, frente al $6.1 \%$ que expresan su total desacuerdo con este pensamiento.

También hemos encontrado que el $91 \%$ de los encuestados están de acuerdo con que la universidad debe poner los medios necesarios para que no sean discriminados, mientras que el $9.1 \%$ afirman su total rechazo hacia esta idea (ítem 3). Concretamente, en el ítem 27, con una media de 3.42 y una desviación típica de 0.56 , podemos observar que el $97 \%$ piensan que se les deben facilitar el acceso a los contenidos y adaptar la metodología y evaluación a sus necesidades, siendo sólo el $45.5 \%$ los que manifiestan estar totalmente de acuerdo con esta idea.

Sin embargo, en referencia al ítem 28, el 57.6\% rechazan la idea de que los contenidos deben ser los mismos que los de sus compañeros y compañeras. Además, el $60.6 \%$ afirman que se debe tener con ellos más flexibilidad a la hora de exigirles la adquisición de las competencias de las titulaciones o carreras (ítem 40). Y lo que es más significativo, como afirman el $81.8 \%$, debe existir profesorado especializado para la atención educativa y social de los estudiantes con discapacidad (ítem 31). En cualquier caso, hay que señalar que existe una alta variabilidad de las distintas respuestas, teniendo en cuenta que el valor de la desviación típica de estos ítems es superior a 1.

Otro de los aspectos significativos a destacar es la existencia de un $100 \%$ de encuestados que se manifiestan en contra tanto de la creencia de que los profesores y profesoras no son capaces de trabajar con ellos (ítem 10), como de la idea de que éstos creen que es difícil trabajar con ellos (ítem 12). Mientras, el 12.2\% piensan que son sus compañeros y compañeras de clase los que no son capaces de trabajar con ellos (ítem 18). En cambio, sólo el $9.1 \%$ de los estudiantes consideran que su discapacidad produce ansiedad a sus compañeros y compañeras de clase (ítem 21), mientras que el $15.2 \%$ creen que es al profesorado a quien le produce ansiedad su discapacidad (ítem 13).

En cualquier caso, sin olvidar la alta desviación típica hallada, hay que señalar que el $48.5 \%$ no creen que se debe informar de ellos mismos a sus compañeros y compañeras de clase (ítem 23). Además, como podemos observar en el ítem 22, el 42.5\% de los encuestados rechazan la idea de que es necesaria la formación/sensibilización de los estudiantes para facilitar su estancia en la universidad. Y lo que es más significativo, el $63.6 \%$ tampoco creen que debe informarse al profesorado acerca de ellos, como estudiantes matriculados en sus asignaturas (ítem 16). 
De otra parte, es importante resaltar que en el ítem 1, con una alta dispersión (0.75) y un grado de desacuerdo significativo (media del 1.48), existe un $15.2 \%$ que piensan que la universidad no es el lugar más adecuado para la formación académica y profesional de los estudiantes con discapacidad. También es necesario señalar que en el ítem 26, con una media de 3.19 y una desviación típica de 0.78 , el $21.2 \%$ no creen que la universidad disponga de los medios para solventar cualquier tipo de adaptación en el acceso al currículum para los estudiantes con discapacidad. Asimismo, en relación con el ítem 30, el $24.3 \%$ de los estudiantes con discapacidad rechazan la idea de que las medidas educativas puestas en marcha por la universidad responden a sus necesidades. Además, el $36.3 \%$ piensan que la evaluación no es respetuosa con su situación y no les asegura la igualdad de oportunidades (ítem 29).

Por último, hay que destacar que el $69.7 \%$ de los encuestados creen que les cuesta más terminar una carrera en la universidad que a los demás compañeros y compañeras (ítem 7), pero no piensan que su discapacidad haya supuesto un obstáculo para sus estudios universitarios (ítem 4). En efecto, como podemos observar en el ítem 2, el $42.4 \%$ están totalmente de acuerdo con que la universidad les pone limitaciones a la hora de acceder a una titulación o carrera. Y lo que es más importante, el $60.6 \%$ no creen que tienen las mismas oportunidades y posibilidades de promoción que los demás estudiantes (ítem 24).

En definitiva, el $63.7 \%$ no comparten la idea de que su integración está asumida por la comunidad universitaria (ítem 37). No obstante, como podemos observar en estos ítems, al igual que en relación con los restantes ítems del cuestionario, existe, en general, una desviación típica alta que supera el valor de 1, lo que indica una alta variabilidad entre las respuestas dadas por los estudiantes con discapacidad en relación con lo expresado en cada uno de ellos. De ahí, la necesidad de completar esta información, analizando las relaciones de contraste entre los factores teóricos y las variables asociadas como son: la edad, el género, centros de estudio y tipo de discapacidad.

Tabla 6. Porcentajes de respuesta, medias y desviaciones típicas del factor actitudes

\begin{tabular}{ccccccc}
\hline ÍTEMS: Factor Teórico Actitudes & 1 & 2 & 3 & 4 & $M$ & $D . T$ \\
\hline 6 & 24.2 & 36.4 & 18.2 & 21.2 & 2.36 & 1.08 \\
36 & 6.1 & 0 & 30.3 & 63.6 & 3.52 & 0.79 \\
\hline
\end{tabular}

En cuanto al factor teórico actitudes, los datos obtenidos indican de forma general un alto grado de compromiso con la integración en la medida en que el $93.9 \%$ de los encuestados afirman que deben conocer las competencias exigidas en cada titulación/carrera antes de matricularse (ítem 36). No obstante, dada la alta desviación típica hallada en este ítem (0.79), hay que destacar que el $6.1 \%$ se expresan en sentido contrario. Además, en relación con el ítem 6, los estudiantes con discapacidad 
manifiestan tener una actitud nada favorecedora hacia le integración, ya que el 39.4\% consideran que preferirían no haber comenzado a realizar los estudios universitarios. En cualquier caso, es necesario señalar que existe una significativa dispersión entre las respuestas con respecto a la media $(M=2.36, D T=1.08)$. Por ello, como hemos comentado con anterioridad, surge la necesidad de realizar un análisis en profundidad de los factores teóricos según las distintas variables asociadas.

\section{Descripción y valoración del ítem abierto del cuestionario}

Bajo la denominación observaciones que desea realizar, el último ítem presenta una estructura abierta, habiendo sido contestado sólo por tres estudiantes con discapacidad. Pero, a pesar de ello, del análisis de las observaciones realizadas por esos estudiantes hemos extraído una información valiosa e interesante.

En cuanto al acceso a la Universidad por parte de los estudiantes con discapacidad, uno de los encuestados es consciente de su importancia no sólo para promover la integración de cualquier persona, con independencia de su situación personal, sino también para fomentar el aprendizaje, cuando afirma que "la Universidad debe ser siempre un espacio de aprendizaje e integración”. Asimismo, en la misma línea, otro de los encuestados concreta señalando la necesidad de desarrollar en la Universidad cualquier tipo de adaptaciones o apoyos partiendo de las características y las necesidades de los estudiantes discapacitados, para facilitar así la realización de sus estudios universitarios de acuerdo con los principios de normalización, integración e individualización ("La Universidad debe tener en cuenta a los alumnos minusválidos para asignarles aulas adaptadas o apoyos adecuados").

En definitiva, los estudiantes con discapacidad deben tener las mismas oportunidades en cuanto a su acceso a la Universidad. No obstante, como afirma otro encuestado, es importante también señalar que "un minusválido ha de saber qué carrera puede y no puede hacer por sus minusvalias", para de este modo, poder realizar sus estudios universitarios de forma apropiada gracias a las adaptaciones pertinentes y, en último término, poder desarrollar sus deberes y responsabilidades profesionales en situaciones adecuadas, contribuyendo así a la plena integración personal, social y laboral.

Por último, hay que señalar también la importancia de tener en cuenta la diversidad que existe en las distintas situaciones de integración, no sólo por parte de los estudiantes con discapacidad, sino también por parte del contexto, como afirma otro encuestado "como todo en la vida no se puede generalizar, y en cuanto al comportamiento de profesores y compañeros tampoco”. 


\section{DISCUSION}

De los análisis realizados podemos destacar las conclusiones que a continuación comentamos, teniendo presente que la extensión y profundidad del estudio permite valorar otras conclusiones no contempladas aquí y que el lector podrá detectar. En términos generales, los estudiantes con discapacidad se manifiestan en contra de la idea de que la Universidad no es el lugar más adecuado para su formación académica y profesional. Además, sólo un $36.3 \%$ están de acuerdo con la idea de que su integración está asumida por la comunidad universitaria. A pesar de ello, existe un grupo importante de estudiantes con discapacidad que no consideran la necesidad de una formación e información sobre su situación, dirigida al personal de la Universidad, imprescindible para su adecuada integración social y educativa y, en concreto, sólo un $69.7 \%$ están de acuerdo con la idea de desarrollar una formación específica del profesorado universitario para trabajar con ellos, y lo que es más significativo, sólo un $36.4 \%$ piensan que debe informarse al profesorado acerca de ellos como estudiantes matriculados en sus asignaturas. En definitiva, existe, en general, un alto grado de desacuerdo sobre la idea de que los profesores y profesoras no son capaces y creen que es difícil trabajar con ellos, así como de que su discapacidad produzca ansiedad a sus compañeros y compañeras de clase.

Existe una tendencia hacia la percepción negativa y al bajo grado de satisfacción por parte de los estudiantes con discapacidad, en general, sobre su integración en la Universidad, en la medida en que un $60.6 \%$ piensan que no tienen las mismas oportunidades y posibilidades de promoción y, sobre todo, cuando un $69.7 \%$ creen que les cuesta más terminar una carrera en la Universidad que a los demás compañeros y compañeras. Además, manifiestan una actitud nada favorecedora, en cuanto que un $39.4 \%$ consideran que preferirían no haber comenzado a realizar los estudios universitarios. Pero esta visión negativa y esta actitud segregadora, propiciadas quizá por la falta de información o por los sentimientos caritativos o por el miedo, cambian, ya que existe una predisposición general al compromiso en pro de la integración educativa y social de los estudiantes con discapacidad en la Universidad, en la medida en que afirman deben conocer las competencias exigidas en cada titulación/carrera antes de matricularse. La mayoría, pues, consideran que la titulación alcanzada por ellos mismos debe ser garante del conjunto de competencias necesarias para su ejercicio profesional y que el $69.7 \%$ manifiestan en contra de la idea de que su discapacidad haya supuesto un obstáculo para sus estudios universitarios.

Existe un acuerdo general con la idea de favorecer una mejora de la atención hacia los estudiantes con discapacidad, para lo que es necesario que la Universidad firme convenios de colaboración con Asociaciones e Instituciones Públicas y/o Privadas. Incluso consideran que en la Universidad debe existir una unidad central que coordine y 
asesore a estudiantes con discapacidad, al profesorado y al personal de administración y servicios. Además, partiendo de la necesidad de asegurar la igualdad de oportunidades en el acceso a la Universidad, manifiestan la necesidad de una normativa específica y de esfuerzos económicos para mejorar la atención educativa que reciben así como que se tienen que fomentar actitudes favorables hacia ellos. En definitiva, consideran que la Universidad debe poner los medios necesarios para que no sean discriminados y, además, se les deben facilitar el acceso a los contenidos y adaptar la metodología y evaluación a sus necesidades. En este sentido, pues, subyace la aceptación de la diversidad humana como valor educativo.

\section{REFERENCIAS}

Consejo de Europa (1996). Carta Social Europea http://ec.europa.eu/archives/europeancouncil/index_es.htm. (Consultado: 10/9/2008).

Decreto 167/2003, de 17 de junio, por el que se establece la ordenación de la atención educativa a los alumnos y alumnas con necesidades educativas especiales asociadas a condiciones sociales desfavorecidas (BOJA, 118 de 23/6/2003).

Fox, D. J. (1981). El Proceso de investigación en educación. Pamplona: EUNSA.

Ley 1/1999 de 31 de marzo, de Atención a las Personas con Discapacidad en Andalucía. (BOJA, 45 de $17 / 4 / 1999$

Ley 13/1982, de 7 de abril, de Integración Social de los Minusválidos. B.O.E. núm. 10330 de abril de 1982.

LEY 17/2007, de 10 de diciembre, de Educación de Andalucía (BOJA, 252 de 26/12/2007)

LEY 51/2003, de 2 de diciembre, de igualdad de oportunidades, no discriminación y accesibilidad universal de las personas con discapacidad (BOE, 03/12/2003).

Ley 6/2001, de 21 de diciembre, de Universidades (BOE, 24/12/2001).

Ley 9/1999, de 18 de noviembre, de Solidaridad en la Educación. (BOJA, 2/12/1999)

Ley Orgánica 1/1990, de 3 de octubre, de Ordenación General del Sistema Educativo (BOE, $238 / 90$ de $4 / 10 / 1990)$.

Ley Orgánica 2/2006, de 3 de mayo, de educación (BOE, 4/5/2006)

Ley Orgánica 9/1995, de 20 de noviembre, de la Participación, la Evaluación y el Gobierno de los Centros Docentes. (BOE, 278 de 21/11/1995).

MEC (1978) Plan Nacional para la Educación Especial http://www.slideshare.net/UPN081/programa-nacional-de-fortalecimiento-a-la-educacinespecial-y-la-integracin-educativa (Consultado: 10/9/2008).

ONU (1983). Programa de Acción Mundial para las Personas con Discapacidad. ONU: http://www.inclusion-ia.org/espa\%F1ol/Norm/onu-docs.htm. (Consultado: 20/10/2008).

ONU (1993). Normas Uniformes sobre la Igualdad de Oportunidades de las Personas con Discapacidad. ONU: http://www.inclusion-ia.org/espa\%F1ol/Norm/onu-docs.htm. (Consultado: 20/10/2008).

Orden de 19/9/2002, por la que se regula el periodo de formación para la transición a la vida adulta y laboral, destinado a jóvenes con necesidades educativas especiales. (BOJA, 125 de 26/10/2002).

Orden de 19/9/2002, por la que se regula la elaboración del Proyecto Curricular de los Centros Específicos de Educación Especial y de la programación de las aulas específicas de Educación Especial en los centros ordinarios. (BOJA, 125 de 26/10/2002). 
Orden de 19/9/2002, por la que se regula la realización de la evaluación psicopedagógica y el dictamen de escolarización. (BOJA, 125 de 26/10/2002).

Real Decreto 334/1985, de 6 de Marzo, de Ordenación de la Educación Especial. (BOE, $16 / 3 / 1985)$.

Real Decreto 696/1995, de 28 de abril, de la Ordenación de la Educación de los Alumnos con Necesidades Educativas Especiales. (BOE, 2/6/1995).

Tratado de la Unión Europea (Amsterdam, 1997). http://ec.europa.eu/archives/europeancouncil/index_es.htm. (Consultado: 10/9/2008).

ANEXO I. Cuestionario

\section{CUESTIONARIO DIRIGIDO A ESTUDIANTES (CON DISCAPACIDAD) DE LA UNIVERSIDAD DE ALMERÍA}

Pretendemos conocer las ideas y actitudes de la comunidad educativa de la Universidad de Almería hacia la integración educativa y social de los estudiantes con discapacidad.

Estamos muy interesados en conocer su respuesta ya que nos facilitará datos de extraordinaria importancia para poner en marcha acciones concretas que mejoren la atención educativa en la comunidad universitaria.

\section{GRACIAS POR SU COLABORACIÓN}

\section{DATOS}

Edad ......Género: Hombre Mujer Titulación/Carrera que cursa.

Curso.......... Tipo de discapacidad (Tache con una $\mathrm{X}$ la respuesta elegida)

Física Psíquica Auditiva Otras

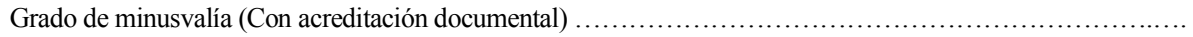

\section{CUESTIONARIO}

CON RESPECTO A LOS ESTUDIANTES DE LA UNIVERSIDAD DE ALMERÍA QUE PRESENTAN ALGÚN TIPO DE DISCAPACIDAD PIENSO QUE:

(Tache con una $\mathrm{x}$ la puntuación más adecuada en función del grado de acuerdo-desacuerdo):
1.- Totalmente en desacuerdo
3.- De acuerdo
2.- En desacuerdo
4.- Totalmente de acuerdo

\begin{tabular}{|c|c|c|c|c|c|}
\hline 1 & $\begin{array}{l}\text { La Universidad no es el lugar más adecuado para la formación académica y profesional de } \\
\text { los estudiantes con discapacidad }\end{array}$ & 1 & 2 & 3 & 4 \\
\hline 2 & La Universidad nos pone limitaciones a la hora de acceder a una titulación/carrera & 1 & 2 & 3 & 4 \\
\hline 3 & La Universidad debe poner los medios necesarios para que no seamos discriminados & 1 & 2 & 3 & 4 \\
\hline 4 & Mi discapacidad ha supuesto un obstáculo para mis estudios en la Universidad & 1 & 2 & 3 & 4 \\
\hline 5 & Es necesario asegurar la igualdad de oportunidades en el acceso a la Universidad & 1 & 2 & 3 & 4 \\
\hline 6 & Preferiría no haber comenzado a realizar los estudios universitarios & 1 & 2 & 3 & 4 \\
\hline 7 & $\begin{array}{l}\text { Nos cuesta más terminar una carrera en la Universidad que a los demás compañeros y } \\
\text { compañeras }\end{array}$ & 1 & 2 & 3 & 4 \\
\hline 8 & No podemos culminar cualquier titulación universitaria & 1 & 2 & 3 & 4 \\
\hline 9 & El profesorado tiene dificultades para relacionarse conmigo & 1 & 2 & 3 & 4 \\
\hline
\end{tabular}




\begin{tabular}{|c|c|c|c|c|c|}
\hline 10 & Los profesores y profesoras no son capaces de trabajar conmigo & 1 & 2 & 3 & 4 \\
\hline 11 & $\begin{array}{l}\text { Los profesores y profesoras tienden a sentir pena por nuestra situación y a ayudarnos en } \\
\text { exceso sin valorar nuestras posibilidades }\end{array}$ & 1 & 2 & 3 & 4 \\
\hline 12 & Los profesores y profesoras creen que es difícil trabajar conmigo & 1 & 2 & 3 & 4 \\
\hline 13 & Mi discapacidad produce ansiedad al profesorado & 1 & 2 & 3 & 4 \\
\hline 14 & Es importante fomentar actitudes favorables hacia nosotros y nosotras & 1 & 2 & 3 & 4 \\
\hline 15 & $\begin{array}{l}\text { Es necesaria una formación específica del profesorado universitario para trabajar con } \\
\text { nosotros }\end{array}$ & 1 & 2 & 3 & 4 \\
\hline 16 & $\begin{array}{l}\text { Debe informarse al profesorado con anticipación de los estudiantes con discapacidad } \\
\text { matriculados en sus asignaturas }\end{array}$ & 1 & 2 & 3 & 4 \\
\hline 17 & Mis compañeros y compañeras de clase tienen dificultades para relacionarse conmigo & 1 & 2 & 3 & 4 \\
\hline 18 & Mis compañeros y compañeras de clase no son capaces de trabajar conmigo & 1 & 2 & 3 & 4 \\
\hline 19 & $\begin{array}{l}\text { Mis compañeros y compañeras de clase tienden a sentir pena por nuestra situación y a } \\
\text { ayudarnos en exceso sin valorar nuestras posibilidades }\end{array}$ & 1 & 2 & 3 & 4 \\
\hline 20 & Mis compañeros y compañeras de clase creen que es difícil trabajar con nosotros & 1 & 2 & 3 & 4 \\
\hline 21 & Mi discapacidad produce ansiedad a los compañeros y compañeras de clase & 1 & 2 & 3 & 4 \\
\hline 22 & $\begin{array}{l}\text { Es necesaria la formación/sensibilización de los estudiantes para facilitar nuestra estancia en } \\
\text { la Universidad }\end{array}$ & 1 & 2 & 3 & 4 \\
\hline 23 & $\begin{array}{l}\text { Debe informarse a los compañeros y compañeras de clase de los estudiantes con } \\
\text { discapacidad matriculados }\end{array}$ & 1 & 2 & 3 & 4 \\
\hline 24 & Tenemos las mismas oportunidades y posibilidades de promoción que los demás estudiantes & 1 & 2 & 3 & 4 \\
\hline 25 & $\begin{array}{l}\text { El profesorado tiene en cuenta la existencia de estudiantes con discapacidad en el aula para } \\
\text { realizar las adaptaciones que sean necesarias }\end{array}$ & 1 & 2 & 3 & 4 \\
\hline 26 & $\begin{array}{l}\text { La Universidad dispone de los medios para solventar cualquier tipo de adaptación en el } \\
\text { acceso al currículum para los estudiantes con discapacidad }\end{array}$ & 1 & 2 & 3 & 4 \\
\hline 27 & $\begin{array}{l}\text { Se nos debe facilitar el acceso a los contenidos y adaptar la metodología y evaluación a } \\
\text { nuestras necesidades }\end{array}$ & 1 & 2 & 3 & 4 \\
\hline 28 & Los contenidos deben ser los mismos que los de nuestros compañeros y compañeras & 1 & 2 & 3 & 4 \\
\hline 29 & La evaluación es respetuosa con nuestra situación y nos asegura la igualdad de oportunidades & 1 & 2 & 3 & 4 \\
\hline 30 & $\begin{array}{l}\text { Las medidas educativas puestas en marcha por la Universidad responden a nuestras } \\
\text { necesidades }\end{array}$ & 1 & 2 & 3 & 4 \\
\hline 31 & $\begin{array}{l}\text { Debe existir profesorado especializado para la atención educativa y social de los estudiantes } \\
\text { con discapacidad }\end{array}$ & 1 & 2 & 3 & 4 \\
\hline 32 & $\begin{array}{l}\text { Debe existir una unidad central que coordine y asesore a estudiantes con discapacidad, al } \\
\text { profesorado y al personal de administración y servicios }\end{array}$ & 1 & 2 & 3 & 4 \\
\hline 33 & $\begin{array}{l}\text { Es necesario que la Universidad firme convenios de colaboración con Asociaciones e } \\
\text { Instituciones Públicas y/o Privadas para nuestra mejor atención }\end{array}$ & 1 & 2 & 3 & 4 \\
\hline 34 & $\begin{array}{l}\text { Debe existir una normativa específica para la atención de los estudiantes con discapacidad en } \\
\text { la Universidad }\end{array}$ & 1 & 2 & 3 & 4 \\
\hline 35 & $\begin{array}{l}\text { La titulación alcanzada por los estudiantes con discapacidad debe ser garante del conjunto de } \\
\text { competencias necesarias para su ejercicio profesional }\end{array}$ & 1 & 2 & 3 & 4 \\
\hline 36 & $\begin{array}{l}\text { Debemos conocer las competencias exigidas en cada titulación/carrera antes de } \\
\text { matricularnos }\end{array}$ & 1 & 2 & 3 & 4 \\
\hline 37 & Nuestra integración está asumida por la comunidad universitaria & 1 & 2 & 3 & 4 \\
\hline 38 & Es necesario que la Universidad desarrolle un plan de acogida para nosotros & & & & \\
\hline 39 & $\begin{array}{l}\text { Se deben incrementar los esfuerzos económicos para mejorar la atención educativa que } \\
\text { recibimos. }\end{array}$ & 1 & 2 & 3 & 4 \\
\hline \multirow[t]{2}{*}{40} & $\begin{array}{l}\text { Se debe tener con nosotros más flexibilidad a la hora de exigirnos la adquisición de las } \\
\text { competencias de las titulaciones/carreras }\end{array}$ & 1 & 2 & 3 & 4 \\
\hline & OBSERVACIONES QUE DESEE FORMULAR & & & & \\
\hline
\end{tabular}

Recibido: 4 de enero de 2010

Recepción Modificaciones: 19 de agosto de 2010 Aceptado: 29 de septiembre de 2010 